Article

\title{
Topological Corner Modes in Graphene-Coated Nanowire Waveguide
}

\author{
Ningliang Liu ${ }^{1}$, Chizhu Ding ${ }^{1}$, Qing Liao ${ }^{2}$ and Shaolin $\mathrm{Ke}^{2, *}$ \\ 1 College of Science, Huazhong Agriculture University, Wuhan 430070, China; \\ liuningliang@mail.hzau.edu.cn (N.L.); dingchizhu@mail.hzau.edu.cn (C.D.) \\ 2 Hubei Key Laboratory of Optical Information and Pattern Recognition, Wuhan Institute of Technology, \\ Wuhan 430205, China; liaoqing@wit.edu.cn \\ * Correspondence: keshaolin@wit.edu.cn
}

Received: 7 April 2020; Accepted: 4 June 2020; Published: 10 June 2020

check for updates

\begin{abstract}
We study the plasmonic second-order topological modes in nanowire waveguides which are covered by monolayer graphene. The waveguide arrays are arranged in the kagome lattice. In a triangle-shaped lattice, the topological modes are localized at the corner of the triangle by tuning the spatial spacing between the different waveguides. The corner modes also depend on the corner shape, which only exist at one corner in a rhombic lattice. In addition to the corner modes, both structures also support the topological edge mode. We show that the corner modes experience a smaller modal wavelength, longer propagation distance, and smaller mode volume than the edge modes. The study may be utilized to explore the topological bound modes at the nanoscale.
\end{abstract}

Keywords: plasmonics; graphene; corner modes

\section{Introduction}

Surface plasmon polaritons (SPPs) are evanescent surface waves that are strongly confined at the interface between a metal and dielectric [1-6]. They are considered as a promising approach to design a nanocircuit beyond the diffraction limit because the effective modal wavelength of SPPs can be squeezed to be much smaller than that in free space $[2,3]$. SPPs are also able to enhance light-matter interactions in aid of strong local field enhancement, which are widely utilized in sensing application and nonlinear optics [1,6]. Recently, graphene, a single-layer carbon atom, has been a hot research topic thanks to its unique band structure and optical properties [7-14]. In the visible spectrum, the optical response of graphene is similar to that of dielectric and shows a constant absorption of $2.3 \%$ of normal illumination [6]. In contrast, graphene behaves like a noble metal and is able to support SPPs in spectra with a larger wavelength, almost covering $\mathrm{THz}$ and infrared frequencies [8]. Moreover, the SPPs of graphene experience stronger field confinement compared with that in metal. The optical properties of SPPs can be flexibly changed through the graphene chemical potential, which is not easily done in traditional bulk metals [13]. In addition, graphene is widely utilized to incorporate with other photonic devices in aid of its excellent mechanical robustness [7,13]. Plasmonic structures based on graphene have been widely explored, such as absorber [15], metamaterial [16], sheet array [17], and nanowire waveguides [3]. Graphene is also a promising nonlinear optical material in multiple nonlinear plasmonics applications, such as optical bistability [18,19], electronic scattering [20], and nonlinear generation of SPPs [21]. Graphene-covered nanowires are also utilized to design waveguide couplers and explore optical discrete and vector soliton in aid of the strong field confinement and huge nonlinear effects of graphene [3,22].

On the other hand, topological photonics provide a new possibility for the study of photonics and have become one the most important research issues in recent years [23-32]. The topological bound 
modes emerge at the boundary that is one dimension lower than that of the bulk when the bulk topology of the system is non-trivial. Such a picture is the first-order of topological insulators and obeys the general bulk-edge correspondence [23]. The topological insulators based on graphene plasmonics are investigated in various platforms [33-39]. The Su-Schriefer-Heeger (SSH) model is proposed in binary graphene sheet arrays $[36,37]$. The Jackiw-Rebbi states are investigated in graphene sheets by changing the chemical potential of graphene [38]. The infrared topological insulator and one-way edge state are reported in periodically patterned monolayer graphene which exhibits quite a wide band gap and strong interaction in THz spectra [39]. However, the previous study of SPPs in graphene are limited to first-order topological bound modes without studying high-order topology. Higher-order topological insulators are a newly predicted topological phase which follow a different topological bulk-boundary correspondence [40-51]. The $(d-n)$-dimensional mode is found to be localized at the boundary of the $n$ th-order of a $d$-dimensional topological insulator. For instance, a second-order topological insulator in a 2D system has topologically protected zero-dimensional corner states instead of 1D topological edge states, which is the common case in standard 2D systems [44,45]. The corner states are also reported in various $3 \mathrm{D}$ structures which are figured out to have a third-order topology. In addition to edge and surface states, the modes that are located at corners can also remain quite robust, which enriches the study of topological states. The corner modes that are revealed in quadrupole insulators are extremely stable even when the boundary of the structure is tremendously reshaped [48]. Topological corner modes are studied in many different platforms, such as electric circuits [41], microwave circuits [42,43], acoustic metamaterials [44], photonic crystal [45,47], plasmon-polaritonic crystals [46], and photonic ring resonators [48].

In this work, we explore the corner modes in a new platform that is constituted of graphene-coated nanowire waveguides. The waveguides are arranged in a breathing kagome lattice, including the triangle and rhombic nanowires. The topological aspect of the system and the related corner modes in this kind of lattice are originally proposed in [49,50], using tight binding models, and have been very recently experimentally realized in acoustic resonators [44] and laser written waveguide arrays [51]. The existence of corner modes is not caused by quadrupole polarization but as a consequence of nonvanishing Wannier centers $[49,50]$. Here, we numerically calculated the effective mode index and the related electric field distributions of corner modes in graphene-coated nanowire arrays. The existence of corner states seriously relay on the relative value of the spatial spacing between the different nanowires. The triangle waveguides have three equivalent corners and the corresponding corner modes are with the degenerate mode index and tend to localize at all corners. In contrast, even the rhombic waveguides have more corners, and there is only a single corner mode located at one corner. We also compare the eigenvalues and mode distributions of the corner and edge modes. The performance of the corner modes is better than the edge modes in the sense that the corner modes encounter a smaller modal wavelength, longer propagation distance, and smaller mode volume than the edge modes.

\section{Triangle Lattice}

We start by studying the corner modes in the triangle structure, which is illustrated in Figure 1. The nanowire waveguide is placed in a triangle lattice and the SPP propagation direction is the $z$ axis. Each unit cell carries three nanowires and the diameter of the nanowire is denoted as $2 a$. Each nanowire is coated by monolayer graphene, which can be realized by the CVD (chemical vapor deposition) growth. The structure has two different spatial spacings, which are denoted by $d_{1}$ and $d_{2}$, respectively. The relative permittivity of the background dielectric is represented by $\varepsilon_{\mathbf{b}}$, which is assumed to be air with $\varepsilon_{\mathrm{b}}=1$ in this study. The nanowire is made up of $\mathrm{SiO}_{2}$ with the permittivity being $\varepsilon_{\mathrm{d}}=3.9$ in the infrared frequency. As a 2D material, the optical material property of graphene depends on surface conductivity $\sigma$. In contrast to bulk metal, the graphene surface conductivity can be adjusted as desired. The surface conductivity $\sigma(T, \omega, \mu, \tau)$ is figured out according to the Kubo formula [6], including inter- and intra-band transitions. The electronic band structures of graphene will be affected 
when graphene is rolled [52,53]. The geometric potential is introduced and the chemical potential of the curved graphene monolayer can be expressed by $\mu_{\mathrm{c}}{ }^{\prime}=\mu_{\mathrm{c}}-\hbar \mathrm{v}_{\mathrm{F}} / \mathrm{a}$, where $\mu_{\mathrm{c}}$ is the chemical potential of monolayer graphene sheet, $\mathrm{v}_{\mathrm{F}} \approx \mathrm{c} / 300$ is the Fermi velocity, and $a$ is the curvature radius. The Kubo formula can be still used with a modified chemical potential. The chemical potential will decrease when the bending is considered. The main conclusions of our manuscript are still valid except that the effective refractive index of SPPs becomes larger.

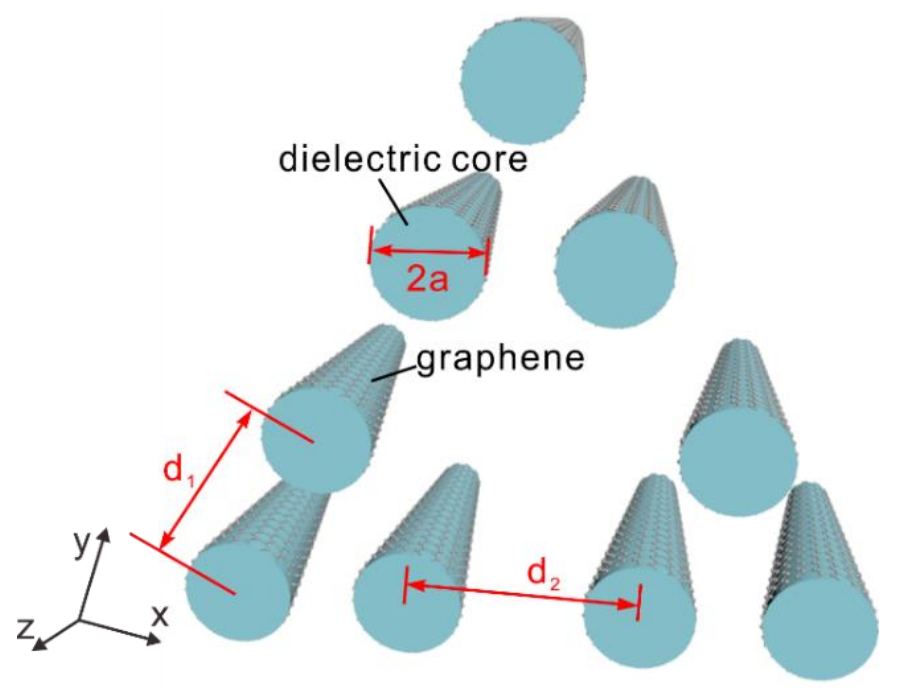

Figure 1. Geometry of the proposed triangular nanowire waveguide.

In this work, we calculate the effective mode index and modal distributions of supermodes using the finite element method [6]. The current that applies in graphene is $\mathbf{j}=\sigma \mathbf{E}$ with $\mathbf{E}$ denoting the electric field vector. Some simulating parameters are chosen as follows and remain unchanged, $\mathrm{T}=300 \mathrm{~K}$, $\mu=0.15 \mathrm{eV}, \tau=0.5 \mathrm{ps}$, and $\lambda=12 \mu \mathrm{m}, a=40 \mathrm{~nm}$. The parameters are tuned in advance such that each graphene nanowire is a single mode waveguide of the SPPs. The effective mode index of the SPP mode in isolated nanowire is almost $n_{\text {eff }} \approx 20$. The coupled mode theory can be used to perform the semi-empirical simulations. The effective refractive index of a single nanowire can be analytically calculated. The coupling strength can be extracted from a double-nanowire coupler. Then, the effective refractive index of the nanowire array can be calculated by the coupled mode theory. Here, we mainly show the results obtained by a full wave simulation.

Topological bound modes depend on the bulk topology, which appears at the boundary of a structure with a nontrivial bulk topology. The structure shown in Figure 1 is similar to the extended $\mathrm{Su}-\mathrm{Schrieffer-Heeger} \mathrm{(SSH)} \mathrm{model} \mathrm{in} \mathrm{2D} \mathrm{arrays,} \mathrm{whose} \mathrm{topology} \mathrm{is} \mathrm{completely} \mathrm{governed} \mathrm{by} \mathrm{the} \mathrm{relative}$ value of the coupling strength between the different nanowires [42]. The system topology of the bulk mode is trivial when the spacing $d_{1}$ between the different waveguides in the same cell is smaller than the spacing $d_{2}$ between the different cells. In contrast, the bulk topology is nontrivial as $d_{1}>d_{2}$. Figure 2 plots the effective mode index of all eigenmodes for trivial and nontrivial structures. The total number of nanowire waveguides is $N=45$, corresponding to five layers of a triangle lattice. The three corners of the triangle lattice are identical. In the above two figures in Figure 2, we plot the complex effective mode index for all supermodes as $d_{1}<d_{2}$. The spatial spacing is $d_{1}=400 \mathrm{~nm}$ and $d_{2}=320 \mathrm{~nm}$. The adjacent waveguides are evanescently coupled and the coupling strength is exponentially decreased with the increase in the spatial spacing. The relative coupling strength between adjacent waveguides in the same cell exceeds that between the different cells as $d_{1}<d_{2}$. As a result, the bulk topology of the nanowires should be trivial and its edges and corners will not allow for topological modes. The results show that there is a bandgap in the spectra of the complex effective mode index. However, no modes are allowed in the bandgap. In the lower two figures in Figure 2, we reverse the relative values of $d_{1}$ and $d_{2}$ and the spatial spacing is $d_{1}=320 \mathrm{~nm}$ and $d_{2}=400 \mathrm{~nm}$. The spatial spacing in the same cell 
now exceeds that in the different cells, that is, $d_{1}>d_{2}$. The intra-layer coupling should be weaker than the inter-layer coupling. The bulk topological property of the nanowire now comes to be nontrivial and is able to sustain topological modes at its boundary. The complex spectra of the effective mode index still possess a bandgap. Moreover, there are three degenerate corner modes denoted as red dots, which are with almost the same effective mode index as $n_{\text {eff }}=19.5+0.25 i$. The effective wavelength of SPPs is inversely proportional to $\operatorname{Re}\left(n_{\text {eff }}\right)$. This indicates the oscillating wavelength of the corner states is approximately $1 / 20$ of the incident wavelength. The subwavelength feature originates from the confinement of graphene plasmonics. Along with the corner modes, nanowires also support nine topological edge modes. Their eigenvalues, that is, the effective mode index, are also located at the bandgap, plotted by the green dots in Figure $2 \mathrm{c}, \mathrm{d}$. Results show that $\operatorname{Re}\left(n_{\text {eff }}\right)$ of the corner modes is larger than that of the edge modes, indicating the corner modes would experience a smaller modal wavelength than the edge modes. In addition, the imaginary effective mode index of the three corner modes is smaller that of the edge modes, reflecting that the corner modes experience smaller propagation loss and that the propagation distance of the corner modes is longer than that of the edge modes.
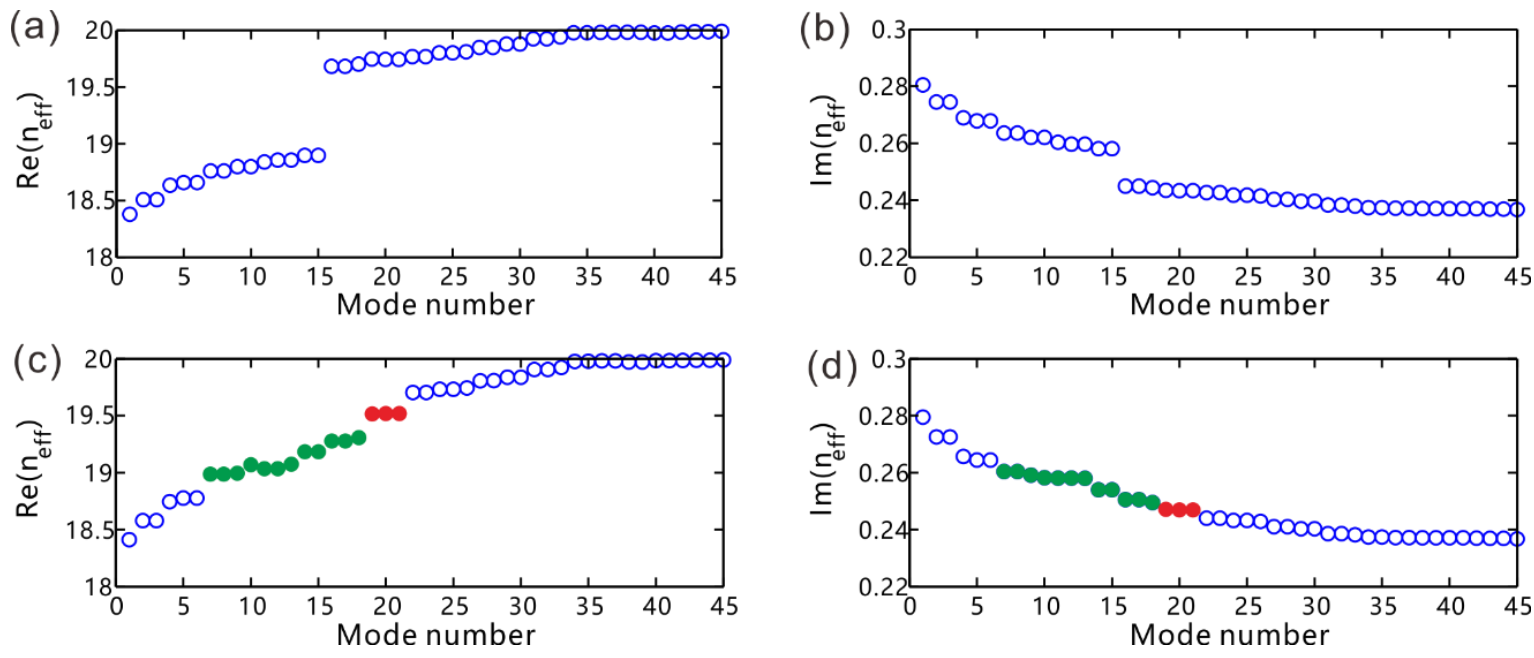

Figure 2. The effective mode index in the triangle nanowires. In $(\mathbf{a}, \mathbf{b})$, the spatial spacing in the nanowires is $d_{1}=400 \mathrm{~nm}$ and $d_{2}=320 \mathrm{~nm}$. In (c,d), the spacing is reversed and $d_{1}=320 \mathrm{~nm}$ and $d_{2}=400 \mathrm{~nm}$. The green dots represent the edge mode while the red dots denote the corner mode.

Although both the edge and corner modes are located in the bandgap, they can be well distinguished from their eigenmodes. Figure 3a-c illustrates the typical profiles of the eigenmodes $(|\mathbf{E}|)$ of the topological corner mode, topological edge modes, and bulk modes, respectively. The corresponding mode indexes are chosen as $n=18,17$, and 45 . In Figure 3a, the field is mainly restricted to the three corners of the triangle and rapidly declines away from the corners. The waveguides at the three corners have the strongest field, the nearest adjacent two waveguides have a vanished field, and the next two waveguides have small fields. This alternating field distribution is also a typical characteristic of the topological mode and guarantees the mode is robust against some disorders. In Figure 3b, the energy is located at the three edges of the triangle and there is almost no energy present in the bulk waveguides nor the waveguides at the three corners. From the field distribution, one can see that the energy of the corner modes is mainly confined at the three waveguides at the corner and field, and the edge modes are extended into many waveguides that are located along the edge. Therefore, the corner modes have a smaller mode volume than the edge modes. In Figure 3c, we plot one case of a bulk mode. The fields almost expand into the entire structure. 

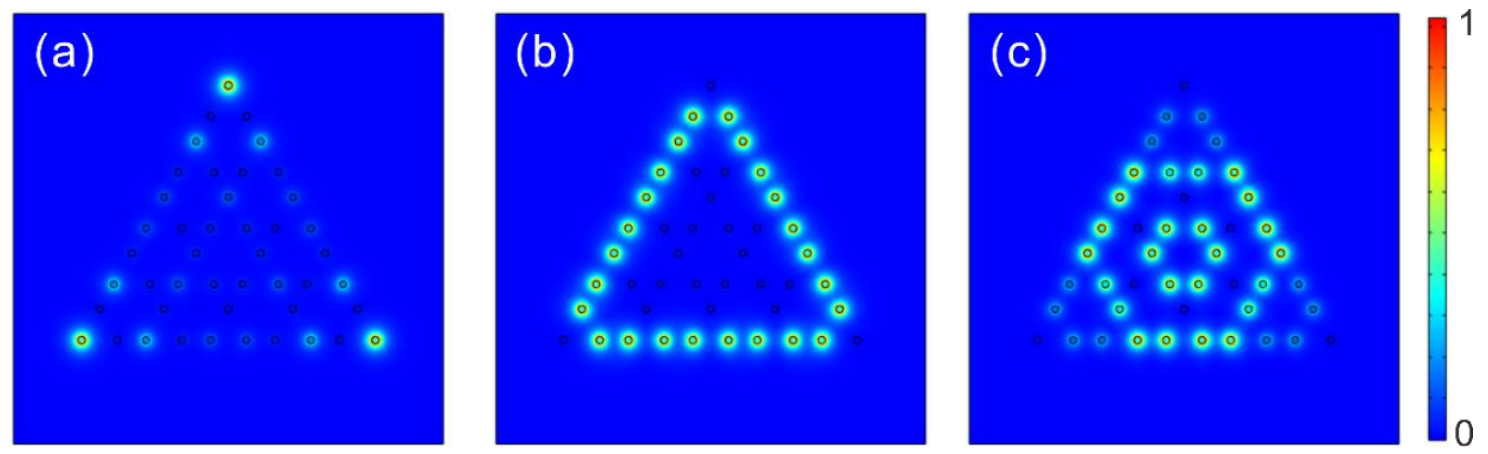

Figure 3. The typical modal profiles (|E|) for the different types of modes. (a) Corner mode, (b) edge mode, and (c) bulk mode.

\section{Rhombic Lattice}

The topological corner modes are determined by the bulk property, which emerge in the structure with a nontrivial bulk topology. Moreover, the corner modes also depend on the corner shape [49]. Some corners are not able to allow for zero modes even if the nanowire is of a nontrivial topology. Such phenomenon is promptly revealed in the rhombic lattice. Figure 4a shows the structure of the graphene-covered nanowires placed in a rhombic lattice. In Figure $4 b, c$, we plot the real and imaginary part of the effective mode index. The whole number of nanowire waveguides is $N=40$. The spectrum of the effective mode index possesses a band gap. One can see the gap only supports a single corner mode, denoted as a red dot. The effective mode index of the corner mode remains unchanged compared with that in a triangle lattice, which is figured out by $n_{\mathrm{eff}}=19.5+0.25 i$. Along with the corner modes, the system also supports six topological edge modes. They are also located at the band gap, denoted by the green dots. Just like the situation in the triangle lattice, the real effective mode index corner mode is larger than that of the edge modes, while $\operatorname{Im}\left(n_{\text {eff }}\right)$ of the corner mode is smaller than the edge mode. Therefore, the corner mode in the rhombic lattice also has a smaller modal wavelength and longer propagation distance than that of the edge modes.
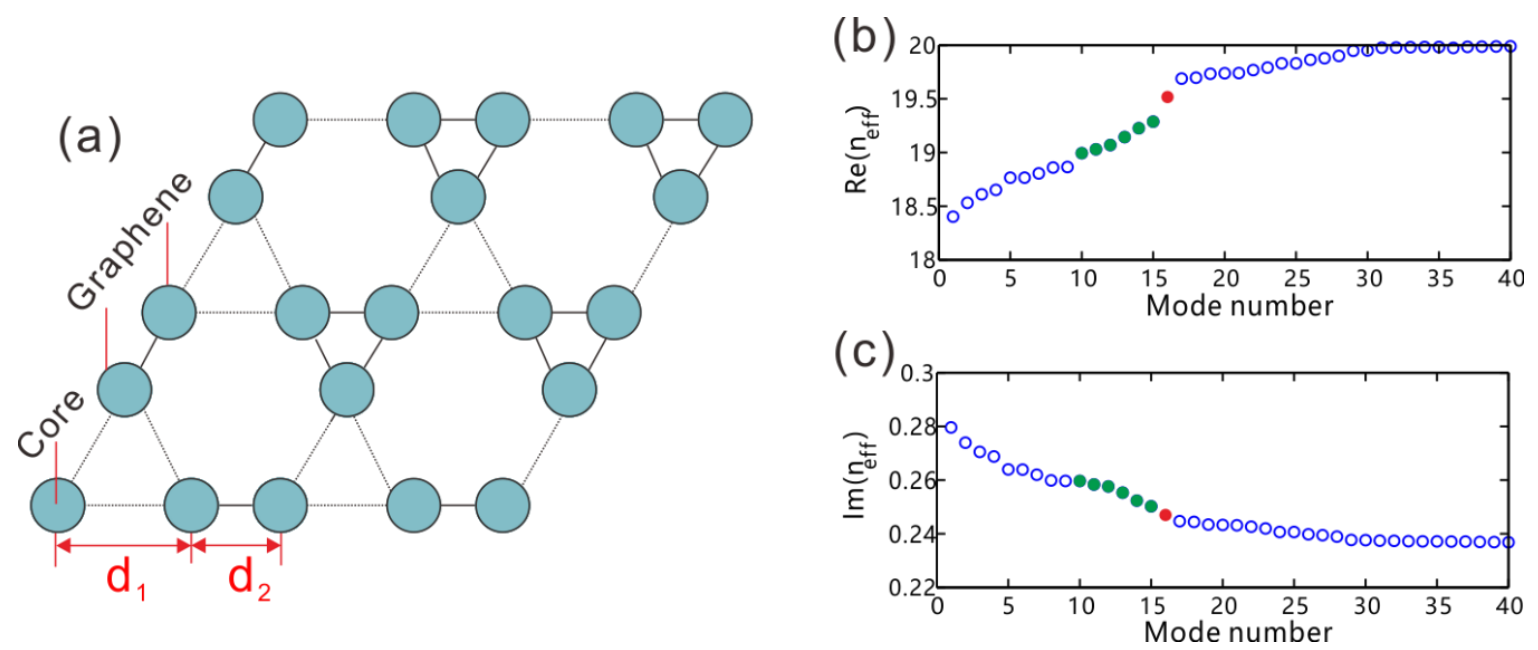

Figure 4. The topological corner mode in a rhombic array. (a) Geometry of a rhombic graphene-coated cylinder waveguide. $(\mathbf{b}, \mathbf{c})$ demonstrate the real and imaginary mode index for $d_{1}=400 \mathrm{~nm}$ and $d_{2}=320 \mathrm{~nm}$.

The discussed triangular lattice has only one type of corner and can support three degenerate corner modes. In contrast, the rhombic lattice has three different types of corners, the lower acute-angled corner, the up acute-angled corner, and two other obtuse-angled corners. The lower acute-angled corner is similar to that in the triangle lattice with a nontrivial topology, while the upper acute-angled 
corner is similar to that in the triangle lattice with a trivial topology. Therefore, one may expect the field will be confined at the lower acute-angled corner. Figure $5 \mathrm{a}, \mathrm{b}$ exhibits the field distributions $(|\mathbf{E}|)$ of one topological edge mode and the topological corner mode, respectively. The corresponding mode indexes are $n=15$ and 16. In Figure 5a, the fields of the edge mode are located at the left and lower edges of the triangle. In Figure $5 \mathrm{~b}$, the energy is mainly confined at the lower acute-angled corner and exponentially decreases away from the corners. There is no energy confined at the two obtuse-angled corners nor the upper trivial acute-angled corner. From the field distribution, one can also see the field of the edge mode is confined in several waveguides while the field of the corner modes mostly is concentrated in the single waveguide at the corner. Therefore, the corner mode has a smaller mode volume than the edge modes.
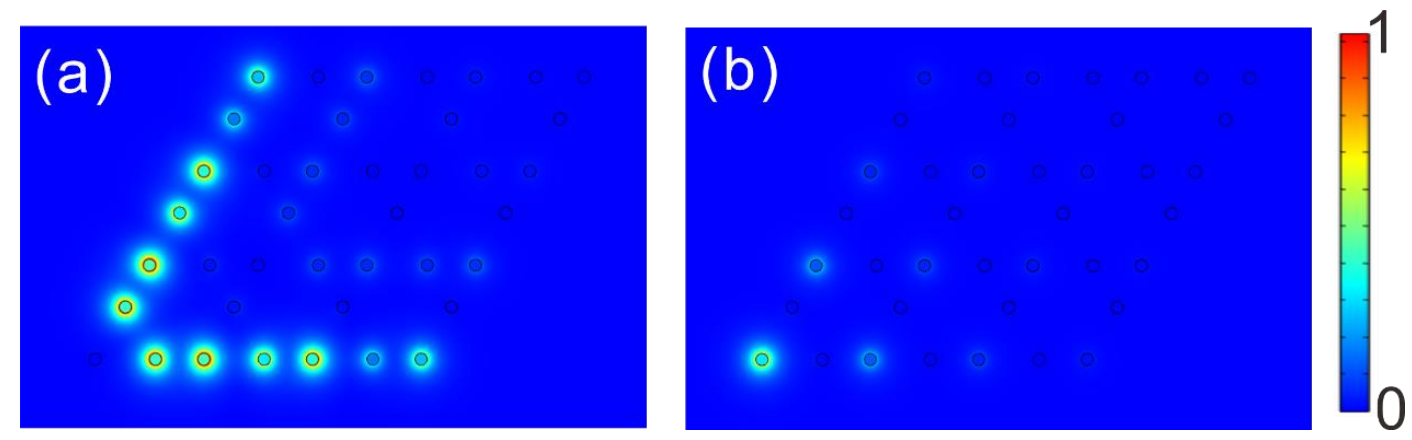

Figure 5. The typical mode profiles $(|\mathbf{E}|)$ in rhombic nanowires. (a) Edge mode and (b) corner mode.

Here, we try to explain the influences of some parameters on the corner mode. The effective refractive index of the graphene-coated nanowire will increase with the decrease in the nanowire radius. The corner mode will persist with different $n_{\text {eff }}$ when the nanowire radius is altered. When the total number of nanowires increases, the corner modes will sustain as well. However, the corner modes may disappear if the number of nanowires is too small. This is because the corner mode is the result of the bulk property. The porosity is mainly determined by the spatial spacing. When the spacing is too large, the band gap will be decreased. The field confinement of the corner modes will become weaker. For small spacing, the nanowires are strongly coupled. The three corner modes will not degenerate and have a different effective refractive index. In [38], the authors discussed the influence of the magnetic field on graphene plasmonics, where the bandgap increases with the increases in the magnetic field. As in our case, we think two effects maybe appear, where the bandgap becomes larger and the three corner modes will not degenerate anymore. The temperature mainly affects the loss of graphene. Therefore, the electric field of distributions of the corner modes will not change. The propagation loss of the corner modes will decrease with the decrease in temperature.

\section{Conclusions}

In summary, we have numerically explored the corner modes of SPPs in 2D arrays constituted of graphene-covered nanowire waveguides. The waveguide arrays are sequenced in a breathing kagome lattice, including the triangle and rhombic lattices. We show the triangle arrays are able to support three corner modes while the rhombic array only supports a single corner mode. The numerical results indicate the corner modes relate to the bulk topology and are determined by the corner shape as well. In a triangle lattice, the structure has three identical corners and can support three degenerate corner modes when the bulk topology is nontrivial. At the same time, the corner modes also depend on the corner shape. In the rhombic waveguide arrays, the nanowire has four corners but the corner modes only exist at one acute-angled corner. Moreover, the edge modes coexist with the corner modes when the bulk topology is nontrivial. Comparing the spectra of the effective mode index and field distributions, we show the corner modes experience a smaller modal wavelength, longer propagation 
distance, and smaller mode volume than the edge modes. Our study enriches the study of high-order topologically protected modes and may be utilized to control light flows beyond the diffraction limit.

Author Contributions: Conceptualization, Q.L. and S.K.; software, N.L. and C.D.; formal analysis, N.L.; investigation, S.K.; writing — original draft preparation, N.L.; writing—review and editing, S.K.; visualization, N.L. and C.D. All authors have read and agreed to the published version of the manuscript.

Funding: Fundamental Research Funds for the Central Universities (2662018JC008), Hubei Provincial Natural Science Foundation of China (2017CFB684), Program for Distinguished Middle-aged and Young Innovative Research Team in Higher Education of Hubei, China (T201806).

Conflicts of Interest: The authors declare no conflict of interest.

\section{References}

1. Maier, S.A. Plasmonics: Fundamentals and Applications; Springer: Berlin, Germany, 2007.

2. Ke, S.; Wang, B.; Qin, C.; Long, H.; Wang, K.; Lu, P. Exceptional points and asymmetric mode switching in plasmonic waveguides. J. Lightwave Technol. 2016, 34, 5258-5262. [CrossRef]

3. Kou, Y.; Förstner, J. Discrete plasmonic solitons in graphene-coated nanowire arrays. Opt. Express 2016, 24, 4714-4721. [CrossRef] [PubMed]

4. Ke, S.; Wang, B.; Long, H.; Wang, K.; Lu, P. Topological mode switching in a graphene doublet with exceptional points. Opt. Quantum Electron. 2017, 49, 1-12. [CrossRef]

5. Wang, Z.; Wang, B.; Long, H.; Wang, K.; Lu, P. Surface plasmonic lattice solitons in semi-infinite graphene sheet arrays. J. Lightwave Technol. 2017, 35, 2960-2965. [CrossRef]

6. Han, X.; Wang, K.; Xing, X.; Wang, M.; Lu, P. Rabi Splitting in a Plasmonic Nanocavity Coupled to a WS2 Monolayer at Room Temperature. ACS Photonics 2018, 5, 3970-3976. [CrossRef]

7. Bao, Q.; Loh, K.P. Graphene photonics, plasmonics, and broadband optoelectronic devices. ACS Nano 2012, 6, 3677-3694. [CrossRef] [PubMed]

8. Zhao, D.; Wang, Z.; Long, H.; Wang, K.; Wang, B.; Lu, P. Optical bistability in defective photonic multilayers doped by graphene. Opt. Quantum Electron. 2017, 49, 163. [CrossRef]

9. Chen, P.Y.; Al, A. Atomically thin surface cloak using graphene monolayers. ACS Nano 2011, 5, 5855-5863. [CrossRef]

10. Ke, S.; Liu, J.; Liu, Q.; Zhao, D.; Liu, W. Strong absorption near exceptional points in plasmonic waveguide arrays. Opt. Quantum Electron. 2018, 50, 318. [CrossRef]

11. Zhao, D.; Liu, F.; Meng, P.; Wen, J.; Xu, S.; Li, Z.; Zhong, D. Reflection Enhancement and Giant Lateral Shift in Defective Photonic Crystals with Graphene. Appl. Sci. 2019, 9, 2141. [CrossRef]

12. Ke, S.; Zhao, D.; Liu, Q.; Liu, W. Adiabatic transfer of surface plasmons in non-Hermitian graphene waveguides. Opt. Quantum Electron. 2018, 50, 393. [CrossRef]

13. Liu, J.X.; Park, S.; Nowak, D.; Tian, M.C.; Wu, Y.; Long, H.; Wang, K.; Wang, B.; Lu, P. Near-Field Characterization of Graphene Plasmons by Photo-Induced Force Microscopy. Laser Photonics Rev. 2018, 2018, 1800040. [CrossRef]

14. Zhao, D.; Xu, B.; Guo, H.; Xu, W.; Zhong, D.; Ke, S. Low Threshold Optical Bistability in Aperiodic PT-Symmetric Lattices Composited with Fibonacci Sequence Dielectrics and Graphene. Appl. Sci. 2019, 9, 5125. [CrossRef]

15. Huang, H.; Ke, S.; Wang, B.; Long, H.; Wang, K.; Lu, P. Numerical study on plasmonic absorption enhancement by a rippled graphene sheet. J. Lightwave Technol. 2017, 35, 320-324. [CrossRef]

16. Li, J.; Fu, J.; Liao, Q.; Ke, S. Exceptional points in chiral metasurface based on graphene strip arrays. J. Opt. Soc. Am. 2019, 36, 2492-2498. [CrossRef]

17. Wang, F.; Qin, C.Z.; Wang, B.; Long, H.; Wang, K.; Lu, P.X. Rabi oscillations of plasmonic supermodes in graphene multilayer arrays. IEEE J. Sel. Top. Quant. 2017, 23, 4600105. [CrossRef]

18. Sanderson, M.; Ang, Y.; Gong, S.; Zhao, T.; Hu, M.; Zhong, R.; Chen, X.; Zhang, P.; Zhang, C.; Liu, S. Optical bistability induced by nonlinear surface plasmon polaritons in graphene in terahertz regime. Appl. Phys. Lett. 2015, 107, 203113. [CrossRef]

19. Zhao, D.; Ke, S.; Hu, Y.; Wang, B.; Lu, P. Optical bistability of graphene embedded in parity-time-symmetric photonic lattices. JOSA B 2019, 36, 1731-1737. [CrossRef] 
20. Ooi, K.; Ang, S.; Cheng, J.L.; Ang, L.K.; Tan, D.T.H. Electronic scattering of graphene plasmons in the terahertz nonlinear regime. IEEE J. Sel. Top. Quantum Electron. 2017, 23, 5100206. [CrossRef]

21. Yao, B.; Liu, Y.; Huang, S.-W.; Choi, C.; Xie, Z.; Flores, J.F.; Wu, Y.; Yu, M.; Kwong, D.-L.; Huang, Y.; et al. Broadband gate-tunable terahertz plasmons in graphene heterostructures. Nat. Photonics 2018, 12, $22-28$. [CrossRef]

22. Xu, S.; Zhou, Q.; Zhao, D.; Belic, M.; Zhao, Y. Spatiotemporal solitons in cold Rydberg atomic gases with Bessel optical lattices. Appl. Math. Lett. 2020, 106, 106230. [CrossRef]

23. Lu, L.; Joannopoulos, J.D.; Soljačić, M. Topological photonics. Nat. Photonics 2014, 8, 821-829. [CrossRef]

24. Ke, S.; Zhao, D.; Liu, J.; Liu, Q.; Liao, Q.; Wang, B.; Lu, P. Topological bound modes in anti-PT-symmetric optical waveguide arrays. Opt. Express 2019, 27, 13858-13870. [CrossRef] [PubMed]

25. Liu, F.; Zhao, D.; Cao, H.; Xu, W.; Ke, S. Exceptional Points in Non-Hermitian Photonic Crystals Incorporated with a Defect. Appl. Sci. 2020, 10, 823. [CrossRef]

26. Deng, H.; Chen, X.; Panoiu, N.C.; Ye, F. Topological surface plasmons in superlattices with changing sign of the average permittivity. Opt. Lett. 2016, 41, 4281-4284. [CrossRef] [PubMed]

27. Fu, N.; Fu, Z.; Zhang, H.; Liao, Q.; Zhao, D.; Ke, S. Topological bound modes in optical waveguide arrays with alternating positive and negative couplings. Opt. Quantum Electron. 2020, 52, 61. [CrossRef]

28. Fu, Z.; Fu, N.; Zhang, H.; Wang, Z.; Zhao, D.; Ke, S. Extended SSH Model in Non-Hermitian Waveguides with Alternating Real and Imaginary Couplings. Appl. Sci. 2020, 10, 3425. [CrossRef]

29. Deng, H.; Chen, Y.; Panoiu, N.C.; Malomed, B.A.; Ye, F. Surface modes in plasmonic Bragg fibers with negative average permittivity. Opt. Express 2018, 26, 2559-2568. [CrossRef] [PubMed]

30. Cheng, Q.; Pan, Y.; Wang, Q.; Li, T.; Zhu, S. Topologically protected interface mode in plasmonic waveguide arrays. Laser Photonics Rev. 2015, 9, 392-398. [CrossRef]

31. Pocock, S.R.; Xiao, X.; Huidobro, P.A.; Giannini, V. Topological plasmonic chain with retardation and radiative effects. ACS Photonics 2018, 5, 2271-2279. [CrossRef]

32. Ling, C.W.; Xiao, M.; Chan, C.T.; Yu, S.F.; Fung, K.H. Topological edge plasmon modes between diatomic chains of plasmonic nanoparticles. Opt. Express 2015, 23, 2021-2031. [CrossRef] [PubMed]

33. Ge, L.; Wang, L.; Xiao, M.; Wen, W.; Chan, C.T.; Han, D. Topological edge modes in multilayer graphene systems. Opt. Express 2015, 23, 21585-21595. [CrossRef] [PubMed]

34. Ke, S.; Wang, B.; Long, H.; Wang, K.; Lu, P. Topological edge modes in non-Hermitian plasmonic waveguide arrays. Opt. Express 2017, 25, 11132-11143. [CrossRef] [PubMed]

35. Meng, P.; Zhao, D.; Zhong, D.; Liu, W. Topological plasmonic modes in graphene-coated nanowire arrays. Opt. Quantum Electron. 2019, 51, 156. [CrossRef]

36. Wang, F.; Ke, S.; Qin, C.; Wang, B.; Long, H.; Wang, K.; Lu, P. Topological interface modes in graphene multilayer arrays. Opt. Laser Technol. 2018, 103, 272-278. [CrossRef]

37. Ge, L.; Liu, L.; Xiao, M.; Du, G.; Shi, L.; Han, D.; Chan, C.T.; Zi, J. Topological phase transition and interface states in hybrid plasmonic-photonic systems. J. Opt. 2017, 19, 1-5. [CrossRef]

38. Xu, C.; Zhang, P.; Zhao, D.; Guo, H.; Huang, M.; Ke, S. Plasmonic Jackiw-Rebbi Modes in Graphene Waveguide Arrays. Appl. Sci. 2019, 19, 4152. [CrossRef]

39. Jin, D.; Christensen, T.; Soljačić, M.; Fang, N.X.; Lu, L.; Zhang, X. Infrared Topological Plasmons in Graphene. Phys. Rev. Lett. 2017, 118, 245301. [CrossRef]

40. Benalcazar, W.A.; Bernevig, B.A.; Hughes, T.L. Quantized electric multipole insulators. Science 2017, 357, 61-66. [CrossRef] [PubMed]

41. Imhof, S.; Berger, C.; Bayer, F.; Brehm, J.W.; Molenkamp, L.; Kiessling, T.; Schindler, F.; Lee, C.; Greiter, M.; Neupert, T.; et al. Topolectrical-circuit realization of topological corner modes. Nat. Phys. 2018, 14, 925-929. [CrossRef]

42. Serra-Garcia, M.; Peri, V.; Süsstrunk, R.; Bilal, O.R.; Larsen, T.; Guillermo, L.; Huber, S.D. Observation of a phononic quadrupole topological insulator. Nature 2018, 555, 342-345. [CrossRef] [PubMed]

43. Peterson, C.W.; Benalcazar, W.A.; Hughes, T.L.; Bahl, G. A quantized microwave quadrupole insulator with topologically protected corner states. Nature 2018, 555, 346-350. [CrossRef] [PubMed]

44. Xue, H.; Yang, Y.; Gao, F.; Chong, Y.; Zhang, B. Acoustic higher-order topological insulator on a kagome lattice. Nat. Mater. 2019, 18, 108-112. [CrossRef] [PubMed]

45. Chen, X.-D.; Deng, W.-M.; Shi, F.-L.; Zhao, F.-L.; Chen, M.; Dong, J.-W. Direct observation of corner states in second-order topological photonic crystal slabs. Phys. Rev. Lett. 2019, 122, 233902. [CrossRef] [PubMed] 
46. Chen, Y.; Lin, Z.; Chen, H.; Jiang, J. Plasmon-polaritonic quadrupole topological insulators. Phys. Rev. 2020, 101, 041109. [CrossRef]

47. Ota, Y.; Liu, F.; Katsumi, R.; Watanabe, K.; Wakabayashi, K.; Arakawa, Y.; Iwamoto, S. Photonic crystal nanocavity based on a topological corner state. Optica 2019, 6, 786-789. [CrossRef]

48. Mittal, S.; Orre, V.; Zhu, G.; Gorlach, M.A.; Poddubny, A.; Hafezi, M. Photonic quadrupole topological phases. Nat. Photonics 2019, 13, 692-696. [CrossRef]

49. Xu, Y.; Xue, R.; Wan, S. Topological corner states on kagome lattice based chiral higher-order topological insulator. arXiv 2017, arXiv:1711.09202.

50. Ezawa, M. Higher-Order Topological Insulators and Semimetals on the Breathing Kagome and Pyrochlore Lattices. Phys. Rev. Lett. 2018, 120, 026801. [CrossRef] [PubMed]

51. Hassan, E.; Ashraf Flore, K.; Kunst, A.M.; Andler, G.; Emil, J.; Bourennane, B.; Bourennane, M. Corner states of light in photonic waveguides. Nat. Photonics 2019, 13, 697-700. [CrossRef]

52. Atanasov, V.; Saxena, A. Tuning the electronic properties of corrugated graphene: Confinement, curvature, and band-gap opening. Phys. Rev. 2010, 81, 205409. [CrossRef]

53. Atanasov, V.; Saxena, A. Electronic properties of corrugated graphene: The Heisenberg principle and wormhole geometry in the solid state. J. Phys. Condens. Matter 2011, 23, 175301. [CrossRef] [PubMed]

(C) 2020 by the authors. Licensee MDPI, Basel, Switzerland. This article is an open access article distributed under the terms and conditions of the Creative Commons Attribution (CC BY) license (http://creativecommons.org/licenses/by/4.0/). 